\title{
Subgel Phase Structure in Monolayers of Glycosylphosphatidylinositol Glycolipids**
}

\author{
Cristina Stefaniu, * Ivan Vilotijevic, Mark Santer, Daniel Varón Silva, Gerald Brezesinski, and \\ Peter H. Seeberger*
}

Glycosylphosphatidylinositols (GPIs) are complex glycolipids that are commonly found in eukaryotic cells as a posttranslational modification of proteins or as free GPIs displayed on the cell surface. ${ }^{[1]}$ Although their main function is to anchor the attached protein (AP) to the cell membrane, ${ }^{[2]}$ the conserved nature of the complex pseudopentasaccharide core of GPIs (Figure 1A) suggests biological roles beyond simple physical anchoring.

The GPI-APs and free GPIs show non-Brownian density fluctuations on cell surfaces, ${ }^{[3]}$ and associate with membrane microdomains, known as lipid rafts. ${ }^{[4]}$ While protein-protein interactions may contribute to the observed clustering of GPI-APs on the cell membranes, ${ }^{[5]}$ such interactions cannot be responsible for clustering of free GPIs and their association with lipid rafts. It is, therefore, conceivable that the interactions between GPI molecules and their interactions with other membrane associated species play a role in the heterogeneous distribution of GPIs in cell membranes. Some proteins that do not form clusters in the cytosol do so on the cell surface when expressed as a GPI-AP construct. ${ }^{[6]}$ Clustering of the free GPIs has also been observed on the cell surface of some parasitic protozoa. ${ }^{[3 c]}$

GPIs and GPI-APs are involved in a variety of biological processes, such as signal transduction, ${ }^{[7]}$ protein sorting and transport,${ }^{[8]}$ intermembrane transfers, ${ }^{[9]}$ parasitic infections,${ }^{[10]}$ and pathophysiology of prion diseases. ${ }^{[11]}$ Insights into the behavior of GPIs and GPI-APs in cell membranes could contribute to the understanding of the roles GPIs play in these processes. ${ }^{[12]}$

[*] Dr. C. Stefaniu, Dr. I. Vilotijevic, Dr. M. Santer, Dr. D. Varón Silva, Prof. Dr. G. Brezesinski, Prof. Dr. P. H. Seeberger

Max Planck Institute of Colloids and Interfaces

Am Mühlenberg 1, 14424 Potsdam (Germany)

E-mail: stefaniu@mpikg.mpg.de seeberger@mpikg.mpg.de

Prof. Dr. P. H. Seeberger

Institute of Chemistry and Biochemistry, Free University of Berlin Arnimallee 22, 14195 Berlin (Germany)

[***] We are grateful to Prof. Dr. Helmuth Möhwald for helpful discussions and Sebastian Götze for providing a building block for synthesis of 1. We thank HASYLAB at DESY, Hamburg (Germany), for beamtime and excellent support. This work was supported by the Max Planck Society. The research leading to these results has received funding from the European Commission's Seventh Framework Programme FP7/2007-2013 (postdoctoral fellowship to I.V.)

Supporting information for this article is available on the WWW under http://dx.doi.org/10.1002/anie.201205825.

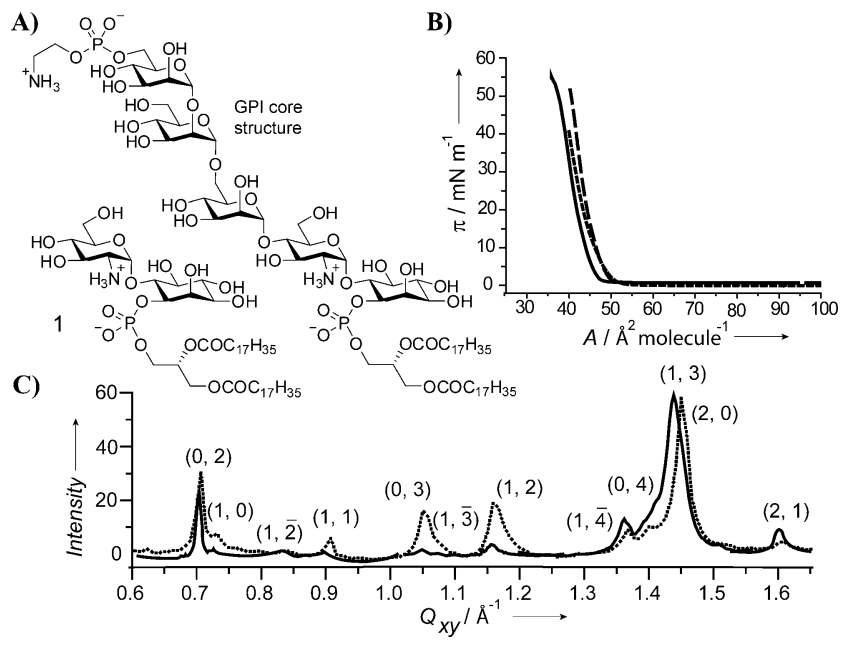

Figure 1. A) Conserved GPI structure and the structure of GlcN $\alpha 1 \rightarrow$ 6 myolno-1-phosphodistearoylglycerol fragment 1. B) Surface pressure/ molecular area $(\pi / A)$ isotherms on the surface of water $(-)$, PBS $(10 \mathrm{~mm}, \mathrm{pH} 7.4,150 \mathrm{~mm} \mathrm{NaCl} ;----)$, and $\mathrm{pH} 2$ solution $(0.01 \mathrm{M} \mathrm{HCl}$; $--)$. C) GIXD patterns of monolayers of 1 on PBS at $20^{\circ} \mathrm{C}\left(2 \mathrm{mN} \mathrm{m}^{-1}\right.$ $\left.\longrightarrow, 30 \mathrm{mN} \mathrm{m}^{-1}-----\right)$.

Monolayers formed with GPI-APs have been investigated ${ }^{[13]}$ but the limited scope did not provide fundamental insights into the behavior of GPIs in cellular membranes. We aim to elucidate the structural characteristics and conformational behavior of GPIs in well-defined membrane models. Herein, we report the unprecedented ordering in two-dimensional monolayers of GlcN $1 \rightarrow 6$ myoIno-1-phosphodistearylglycerol fragment of GPIs (1; Figure 1A) observed by grazing-incidence $\mathrm{X}$-ray diffraction (GIXD).

Synthetic GPI mimic 1 represents a minimal fragment that might adequately emulate GPI behavior. While $\mathbf{1}$ lacks the trimannose portion present in all known GPIs, the glucosaminephosphoinositol moiety features both the amino and phosphate groups largely seen as major determinants of the behavior of the charged head groups.

Compound 1 was studied in 2D films confined at the air/ liquid interface that are easy-to-handle model systems of one membrane leaflet (Figure 1A). The reduced dimensionality of the system is advantageous to better understand the role of different interactions for structure formation. To gain first insights into the molecular interactions and the possible phase transitions of compound $\mathbf{1}$ in monolayers, surface pressure/ molecular area isotherms were recorded on different subphases (Figure 1B). The water subphase was used in the presence and absence of $\mathrm{Ca}^{2+}$ ions to test for differences that 
may arise from the formation of calcium phosphate bridges between the glycophospholipid molecules. ${ }^{[14]}$ Phosphate-buffered saline (PBS; $10 \mathrm{~mm}, \mathrm{pH} 7.4,150 \mathrm{~mm} \mathrm{NaCl}$ ) and a $\mathrm{pH} 2$ $(0.01 \mathrm{M} \mathrm{HCl})$ subphase were used to investigate the differences that may arise from various degrees of ionization of the phosphate group. Besides being slightly shifted, the compression isotherms exhibit no distinct features (Figure 1B), indicating that the monolayers are not significantly influenced by different subphases. In all cases, a sharp increase of the surface pressure was recorded for molecular areas smaller than $48 \AA^{2}$ per molecule, suggesting the transition from a 2D gas phase into a $2 \mathrm{D}$ liquid condensed phase at close to zero surface pressure.

To obtain further structural information related to the proposed phase transition, monolayers of $\mathbf{1}$ were analyzed by GIXD on different subphases. The monolayers of acylglycerols, diacyl-sn-glycerols, and diacyl-sn-phosphatidylinositols exhibit only the Bragg peaks of the hydrophobic chain lattice in the wide-angle region. ${ }^{[15]}$ In contrast, the GIXD patterns of monolayers of $\mathbf{1}$, measured on different subphases, reveal several Bragg peaks in the mid-to-wide angle region (Figure $1 \mathrm{C}$ ). These additional peaks indicate a head-group ordering that was not observed in any of the previous studies on double-chain phospholipids, including phospholipids with head groups that can be engaged in hydrogen-bonding interactions. The positions of Bragg peaks for monolayers of $\mathbf{1}$ are not affected by the identity of the subphase used in the experiments and they shift only slightly upon compression from $2 \mathrm{mN} \mathrm{m}^{-1}$ to $30 \mathrm{mNm}^{-1}$ (Figure $1 \mathrm{C}$ ).

The wide-angle-region reflections that are characteristic for ordered alkyl chains were analyzed in detail. Selected contour plots (Figure 2A) indicate that the monolayer structure is characterized by an oblique (triclinic or mono-

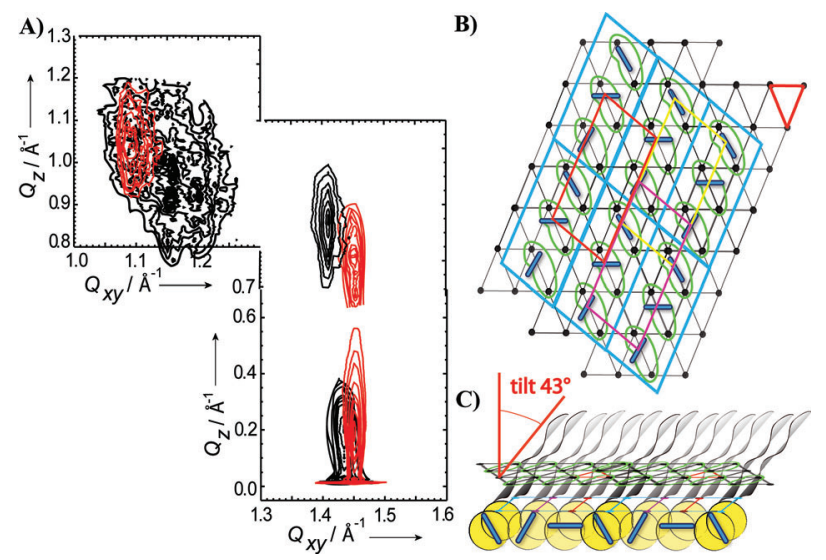

Figure 2. A) Contour plots of corrected $X$-ray intensities as a function of in-plane $\left(Q_{x y}\right)$ and out-of-plane $\left(Q_{z}\right)$ scattering vector components of monolayers of 1 on PBS $\left(2 \mathrm{mN} \mathrm{m}^{-1}\right.$ black —, and $30 \mathrm{mN} \mathrm{m}^{-1}$ red $-)$. B) Representation of the commensurable lattices describing the lateral order of the alkyl chains (black dots; repeating unit cell shown by the red triangle) and of the molecules (repeating unit cell shown by the blue parallelogram). Repeating unit cells (red, yellow, and magenta) link the molecules with the same head group orientation. Delimitation of the molecules in the lattice plane indicated by the green line: view from above. C) Representation of the molecules at the air/water interface: lateral view. clinic) chain lattice (represented as the black dotted lattice in Figure $2 \mathrm{~B}) \cdot{ }^{[16]}$ The Bragg rods are characterized by a value of the full-width at half-maximum (fwhm) of $0.26 \AA^{-1}$. The determined length $L$ of the scattering unit $\left(\mathrm{fwhm}\left(Q_{\mathrm{z}}\right) \approx 0.9\right.$ $(2 \pi) / \mathrm{L})^{[17]}$ is $21.7 \AA$. This value agrees well with the length of an extended $\mathrm{C}_{18}$ alkyl chain in an all-trans conformation. This observation confirms that the interfacial layer is a monolayer at both low and high lateral pressures. While multilayers could also generate additional Bragg peaks, the fwhm of their Bragg rods would be significantly smaller. ${ }^{[18]}$

Indexing of the additional Bragg peaks (Figure 1C) revealed the existence of a supercell that is indicative of the ordering of entire molecules. This ordering is likely a result of strong interactions between the head groups. Numerical analysis $^{[19]}$ reveals that the lipids (green solid lines) are likely positioned as depicted in Figure 2B. The supercell (blue parallelogram) is defined by the lattice parameters $a_{\mathrm{s}}=$ $9.06 \AA, b_{\mathrm{s}}=18.68 \AA$, an angle $\gamma$ of $107.4^{\circ}$ and it maps an area of $161.6 \AA^{2}$ that corresponds to three molecules of $\mathbf{1}$. The monolayer structure is characterized by the ordering of entire molecules and by a larger repeating unit consisting of three lipid molecules. This observation suggests that the three molecules in the supercell feature distinct characteristics such as varying head-group orientations and/or different conformations of the pseudodisaccharide unit. It is plausible that the head groups in $\mathbf{1}$ have to reorient to engage in strong intermolecular interactions. Thus, the supercell can be described by the repeating unit of identically oriented molecules (Figure 2B; three differently colored representations of the same supercell linking four molecules with the same orientation of the head group).

The rigid and highly ordered arrangement of the pseudodisaccharide head groups agrees well with the lack of major changes in the monolayer structure upon lateral compression and with a $43^{\circ}$ tilt angle of the alkyl chains (in respect to the normal to the interface, Figure $2 \mathrm{C}$ ). The strong interactions of the head groups control the molecular packing in invariably rigid monolayers of $\mathbf{1}$. The area requirement of the head group is much larger than that required for two alkyl chains which tilt strongly to maximize their van der Waals interactions.

The similarities between the GIXD patterns obtained for the monolayers of $\mathbf{1}$ prepared on subphases of different $\mathrm{pH}$ values $(2,6$, and 7.4) and ionic strengths (pure water, $2 \mathrm{~mm}$ $\mathrm{CaCl}_{2}$, or $150 \mathrm{~mm} \mathrm{NaCl}$ ) suggest that the interactions between the head groups are not simple electrostatic interactions of the ammonium and/or phosphate groups within the glucosamine and phosphodiester of $\mathbf{1}$ (Figure $3 \mathrm{~A}$ ). In that case, the monolayers of $\mathbf{1}$ would show distinct features on these subphases owing to different degrees of ionization of the head groups or electrostatic interactions with oppositely charged ions that could even bridge the phosphates in the head group region, resulting in tighter packing, as shown for divalent cations. ${ }^{[20]}$

After excluding the simple electrostatic interactions as the reason for head-group ordering, we hypothesized that a complex, defined hydrogen bonding network between the head groups of $\mathbf{1}$ may be responsible for the formation of a molecular lattice in monolayers of $\mathbf{1}$. To test this hypothesis, 


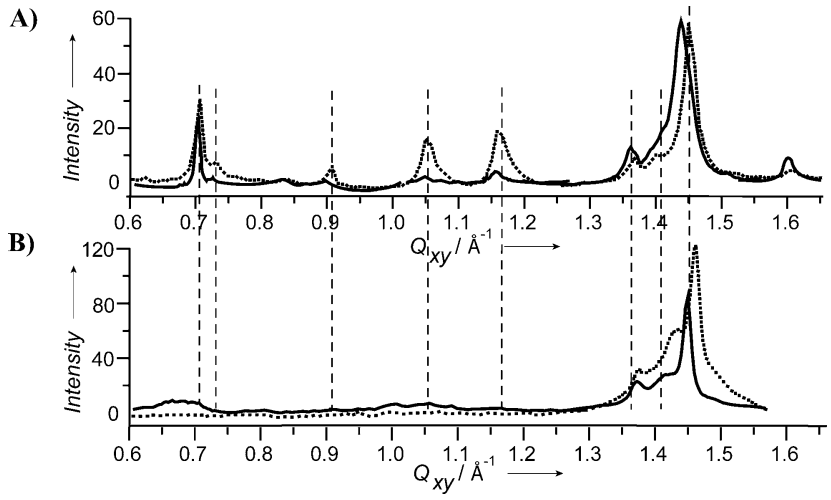

Figure 3. GIXD patterns obtained for 1 on the surface of $A$ ) PBS $(10 \mathrm{~mm}, \mathrm{pH} 7.4,150 \mathrm{~mm} \mathrm{NaCl})$ at $20^{\circ} \mathrm{C}\left(2 \mathrm{mN} \mathrm{m}^{-1}\right.$ and $30 \mathrm{mN} \mathrm{m}^{-1}$-.---) and B) $5 \mathrm{M}$ urea aqueous solution at $20^{\circ} \mathrm{C}\left(2 \mathrm{mN} \mathrm{m}^{-1}\right.$ - and $\left.30 \mathrm{mN} \mathrm{m}^{-1}----\right)$.

monolayers of 1 prepared on the surface of a $5 \mathrm{M}$ urea solution, known for disrupting both inter- and intramolecular hydrogen bonding, ${ }^{[21]}$ were investigated by GIXD. Because urea is not surface-active, comparison of the monolayers on the concentrated urea and water subphases provides a good assessment of the hydrogen-bonding contributions to the interactions between head groups. The GIXD pattern of 1 changes drastically on the urea solution (Figure 3 ). The Bragg peaks characteristic for the condensed state of the alkyl chains shift to larger $Q_{x y}$ values, while the Bragg peaks defining the supercell of the monolayer appear very weak and broad at low surface pressures $\left(2 \mathrm{mN} \mathrm{m}^{-1}\right.$; Figure $\left.3 \mathrm{~B}\right)$ and vanish at high lateral packing densities $\left(30 \mathrm{mN} \mathrm{m}^{-1}\right.$; Figure $3 \mathrm{~B}$ ). The supercell ordering of $\mathbf{1}$ is suppressed on the urea solution subphase, suggesting that hydrogen-bonding interactions are indeed responsible for head-group ordering. This hydrogen-bonding network could originate either from the interactions between the phosphate and the sugar hydroxy groups, ${ }^{[22]}$ from the carbohydrate-carbohydrate interactions between the pseudodisaccharides of $\mathbf{1},{ }^{[23]}$ or from a judicious combination of the two. Several reports describe the effects of urea on the behavior of monolayers that presumably results from disrupting the hydrogen bonding between the head groups. ${ }^{[24]}$ Our results provide the first direct evidence for the changes in the structure of monolayers caused by urea and observed by GIXD.

Urea acts as a chaotropic agent, leading to rotational disorder of the head groups. Consequently, a monolayer of 1 on $5 \mathrm{~m}$ urea subphase only features the lattice of ordered alkyl chains as is common for lipid gel phases. In the absence of the strong rigidifying effect of the head group network on the aqueous urea solution, the molecules are allowed to pack tighter, giving rise to a modified chain lattice (Figure 4) ${ }^{[25]}$ This lattice is defined by Bragg peaks at different $Q_{x y}$ and $Q_{z}$ values compared to those obtained on PBS, showing that the suppression of the head group lattice allows for the alkyl chains to be less tilted $\left(38^{\circ}\right.$; Figure $\left.4 \mathrm{C}\right)$.

To establish the clustering ability of compound $\mathbf{1}$, its miscibility behavior was studied in mixtures with POPC (1palmitoyl-2-oleoyl-phosphatidylcholine), a low-melting model membrane phospholipid. POPC features a common

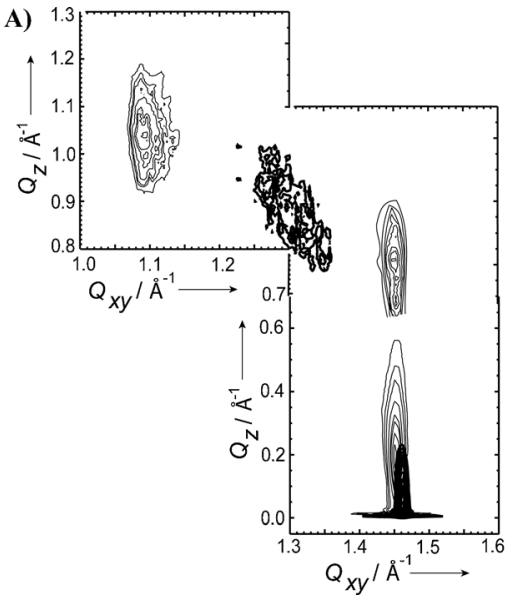

B)

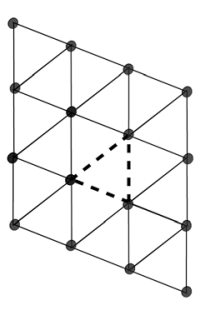

C)

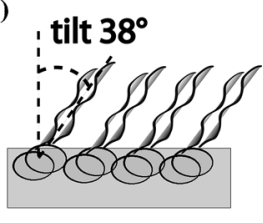

Figure 4. A) Contour plots of corrected X-ray intensities as a function of in-plane $\left(Q_{x y}\right)$ and out-of-plane $\left(Q_{z}\right)$ scattering vector components of monolayers of 1 obtained on PBS $(10 \mathrm{~mm}, \mathrm{pH} 7.4,150 \mathrm{~mm} \mathrm{NaCl}$; $30 \mathrm{mN} \mathrm{m}^{-1}$, thin line pattern) compared to those obtained on the surface of $5 \mathrm{M}$ urea aqueous solutions at $20^{\circ} \mathrm{C}\left(30 \mathrm{mN} \mathrm{m}^{-1}\right.$, thick line pattern). B) Representation of the lattice (chains: black dots; unit cell: dashed line triangle) describing the monolayer structure on the surface of $5 \mathrm{M}$ urea aqueous solutions. C) Representation of the molecules at the air/water interface: lateral view.

hydrophobic motif found in naturally occurring phospholipids, ${ }^{[26]}$ and at $20^{\circ} \mathrm{C}$ it forms monolayers in a liquid disordered state. The experiments were carried out with equimolar mixtures of $\mathbf{1}$ and POPC. Apart from the peaks that are characteristic for pure compound $\mathbf{1}$ (Figure 1C), the GIXD pattern of the mixed monolayer features three additional Bragg peaks (Figure 5; thick line pattern). The appearance of only three additional Bragg peaks indicates that this phase is characterized by a chain lattice with small correlation length and not by a molecular lattice observed in monolayers of $\mathbf{1}$. These results suggest the coexistence of two ordered phases: the solid phase of the pure compound 1 (Figure $5 \mathrm{~A}$; thin line

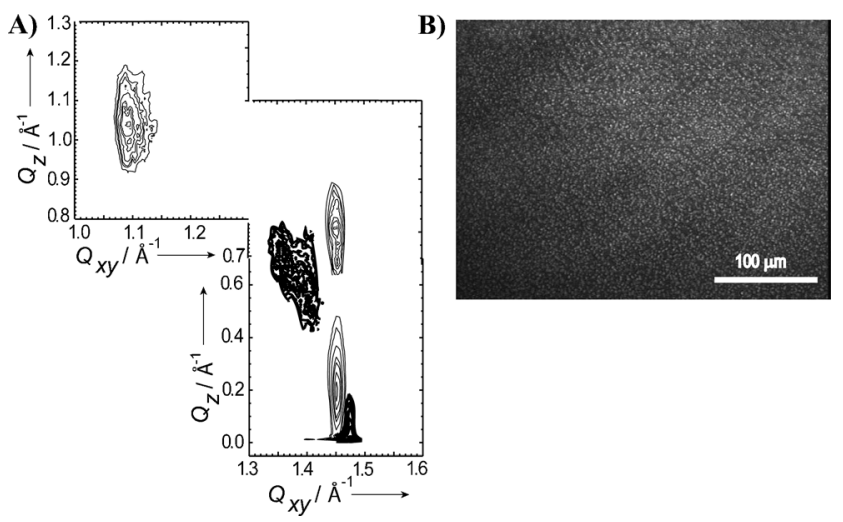

Figure 5. A) GIXD contour plots in the chain lattice region showing the coexistence of two ordered phases: the solid phase of pure compound 1 (thin line pattern) and a liquid ordered phase of mixed 1/POPC (thick line pattern) in monolayers of co-spread mixtures of 1 and POPC (1:1 molar ratio) at $30 \mathrm{mN} \mathrm{m}^{-1}$ on PBS (10 mM, pH 7.4, $150 \mathrm{~mm}$ $\mathrm{NaCl}), 20^{\circ} \mathrm{C}$. B) Brewster-angle microscopy image of the mixed monolayer. The solid phase of the pure compound 1 can be seen as small bright domains surrounded by the mixed liquid ordered phase. 
pattern) and a liquid ordered phase of a 1/POPC mixture. ${ }^{[16]}$ Brewster-angle microscopy confirmed the coexistence of the two phases (Figure 5B).

Based on the GXID analysis, the phase-separated solid domains contain only compound $\mathbf{1}$ with the same crystallographic structure as in the single-component monolayers, suggesting that the strong head-group interactions prohibit the incorporation of the membrane component that forms a disordered phase. When mixed with POPC, compound $\mathbf{1}$ induces ordering in the liquid disordered POPC phase in a cooperative way that is similar to sphingomyelin/POPC mixtures, previously described as representative for a model raft system. ${ }^{[27]}$

In summary, this biophysical study reveals the unprecedented crystalline two-dimensional structure of $\mathrm{GlcN \alpha} 1 \rightarrow$ 6myoIno-1-phosphodistearoylglycerol monolayers. These monolayers are characterized by two commensurate lattices: the oblique lattice of the alkyl chains and the molecular lattice formed owing to highly ordered head groups. This structure is reminiscent of subgel phase ${ }^{[28]}$ structures observed in lipid dispersions after partial dehydration of the head groups during long incubation periods at low temperature. ${ }^{[29]}$ The head-group ordering is observed regardless of incubation period because a hydrogen-bond network rigidifies the monolayer structure. The network of hydrogen bonds is disrupted on highly concentrated urea subphases, leading to the loss of the molecular lattice and the restructuring of the monolayer as observed by GIXD.

Studies on mixed monolayers of $\mathbf{1}$ and POPC demonstrate that above a certain threshold concentration of compound $\mathbf{1}$, phase-separation occurs owing to the strong head-group interactions. Below this threshold concentration, compound 1 mixes with the liquid disordered POPC and induces order in a highly cooperative way. Thus, the GPI fragment $\mathbf{1}$ tends to create ordered phases as it either forms a highly crystalline structure or induces liquid ordered domains (rafts). This ability could have important implications for the interactions of GPI-APs and GPIs in real cell membranes.

Received: July 23, 2012

Revised: September 17, 2012

Published online: November 7, 2012

Keywords: glycosylphosphatidylinositol · hydrogen bonds . lipid rafts - molecular lattices · monolayers

[1] a) M. G. Paulick, C. R. Bertozzi, Biochemistry 2008, 47, 6991; b) M. A. Ferguson, J. Cell Sci. 1999, 112, 2799.

[2] M. Low, A. Saltiel, Science 1988, 239, 268.

[3] a) T. Friedrichson, T. V. Kurzchalia, Nature 1998, 394, 802; b) R. Varma, S. Mayor, Nature 1998, 394, 798; c) N. Azzouz, H. ShamsEldin, S. Niehus, F. Debierre-Grockiego, U. Bieker, J. Schmidt, C. Mercier, M.-F. Delauw, J.-F. Dubremetz, T. K. Smith, R. T. Schwarz, Int. J. Biochem. Cell Biol. 2006, 38, 1914.

[4] a) K. Simons, E. Ikonen, Nature 1997, 387, 569; b) I. Levental, M. Grzybek, K. Simons, Biochemistry 2010, 49, 6305; c) C. Eggeling, C. Ringemann, R. Medda, G. Schwarzmann, K Sandhoff, S. Polyakova, V. N. Belov, B. Hein, C. von Middendorff, A. Schonle, S. W. Hell, Nature 2009, 457, 1159.
[5] S. Paladino, D. Sarnataro, R. Pillich, S. Tivodar, L. Nitsch, C. Zurzolo, J. Cell Biol. 2004, 167, 699.

[6] P. Sharma, R. Varma, R. C. Sarasij, Ira, K. Gousset, G. Krishnamoorthy, M. Rao, S. Mayor, Cell 2004, 116, 577.

[7] K. Simons, D. Toomre, Nat. Rev. Mol. Cell Biol. 2000, 1, 31.

[8] a) M. Fujita, T. Kinoshita, Biochim. Biophys. Acta Mol. Cell Biol. Lipids 2012, 1821, 1050; b) S. Mayor, H. Riezman, Nat. Rev. Mol. Cell Biol. 2004, 5, 110; c) S. Schuck, K. Simons, J. Cell Biol. 2006, $172,963$.

[9] D. Kooyman, G. Byrne, S. McClellan, D. Nielsen, M. Tone, H. Waldmann, T. Coffman, K. McCurry, J. Platt, J. Logan, Science 1995, 269, 89.

[10] a) L. Schofield, M. C. Hewitt, K. Evans, M.-A. Siomos, P. H. Seeberger, Nature 2002, 418, 785; b) N. W. Brattig, K. Kowalsky, X. Liu, G. D. Burchard, F. Kamena, P. H. Seeberger, Microbes Infect. 2008, 10, 885; c) N. Azzouz, F. Kamena, P. H. Seeberger, OMICS 2010, 14, 445.

[11] a) B. Chesebro, M. Trifilo, R. Race, K. Meade-White, C. Teng, R. LaCasse, L. Raymond, C. Favara, G. Baron, S. Priola, B. Caughey, E. Masliah, M. Oldstone, Science 2005, 308, 1435; b) S. P. Mahal, J. Jablonski, I. Suponitsky-Kroyter, A. M. Oelschlegel, M. E. Herva, M. Oldstone, C. Weissmann, PLoS Pathog. 2012, 8, e1002746; c) M. Klingeborn, B. Race, K. D. MeadeWhite, R. Rosenke, J. F. Striebel, B. Chesebro, J. Virol. 2011, 85, 1484.

[12] M. G. Paulick, M. B. Forstner, J. T. Groves, C. R. Bertozzi, Proc. Natl. Acad. Sci. USA 2007, 104, 20332.

[13] a) F. Ronzon, B. Desbat, T. Buffeteau, C. Mingotaud, J.-P. Chauvet, B. Roux, J. Phys. Chem. B 2002, 106, 3307; b) L. Caseli, D. C. Masui, R. P. M. Furriel, F. A. Leone, M. E. D. Zaniquelli, Colloids Surf. B 2005, 46, 248; c) F. Ronzon, J.-P. Rieu, J.-P. Chauvet, B. Roux, J. Colloid Interface Sci. 2006, 301, 493.

[14] W. G. Ellenbroek, Y.-H. Wang, D. A. Christian, D. E. Discher, P. A. Janmey, A. J. Liu, Biophys. J. 2011, 101, 2178.

[15] a) G. Brezesinski, K. de Meijere, H. Möhwald, E. Scalas, W. G. Bouwman, K. Kjaer, Prog. Colloid Polym. Sci. 1996, 100, 351; b) U. Gehlert, D. Vollhardt, G. Brezesinski, H. Möhwald, Langmuir 1996, 12, 4892; c) G. H. Peters, S. Toxvaerd, N. B. Larsen, T. Bjørnholm, K. Schaumburg, K. Kjaer, Nat. Struct. Biol. 1995, 2, 395; d) K. Wagner, G. Brezesinski, Chem. Phys. Lipids 2007, 145, 119.

[16] Details of the lattice parameters are given in the Supporting Information, Tables S1 and S2.

[17] a) K. Kjaer, Phys. B 1994, 198, 100; b) D. Vollhardt, G. Brezesinski, S. Siegel, G. Emrich, J. Phys. Chem. B 2001, 105, 12061.

[18] a) I. Kuzmenko, M. Kindermann, K. Kjaer, P. B. Howes, J. AlsNielsen, R. Granek, G. von Kiedrowski, L. Leiserowitz, M. Lahav, J. Am. Chem. Soc. 2001, 123, 3771; b) H. Rapaport, I. Kuzmenko, S. Lafont, K. Kjaer, P. B. Howes, J. Als-Nielsen, M. Lahav, L. Leiserowitz, Biophys. J. 2001, 81, 2729.

[19] A detailed description of the peak indexing procedure is given in the Supporting Information.

[20] E. Maltseva, V. L. Shapovalov, H. Möhwald, G. Brezesinski, J. Phys. Chem. B 2006, 110, 919.

[21] a) G. E. Hibberd, A. E. Alexander, J. Phys. Chem. 1962, 66, 1854; b) R. B. Simpson, W. Kauzmann, J. Am. Chem. Soc. 1953, 75,5139 .

[22] a) G. Das, A. D. Hamilton, J. Am. Chem. Soc. 1994, 116, 11139; b) G. Das, A. D. Hamilton, Tetrahedron Lett. 1997, 38, 3675.

[23] a) I. Bucior, M. M. Burger, Curr. Opin. Struct. Biol. 2004, 14, 631; b) J. M. de La Fuente, S. Penadés, Glycoconjugate J. 2004, 21, 149 ; c) S. Hakomori, Pure Appl. Chem. 1991, 63, 473; d) N. Seah, A. Basu, Carbohydrate-Carbohydrate Interactions. Wiley Encyclopedia of Chemical Biology, Wiley, Hoboken, 2008, pp. 1-7.

[24] a) I. Levental, A. Cejbers, P. A. Janmey, J. Am. Chem. Soc. 2008, 130, 9025; b) S. M. B. Souza, H. Chaimovich, M. J. Politi, 


\section{Angewandte}

Communications

Langmuir 1995, 11, 1715; c) P. Lo Nostro, G. Gabrielli, Langmuir 1993, 9, 3132.

[25] See the Supporting Information, Tables S1 and S2.

[26] D. Marsh, Handbook of Lipid Bilayers, CRC, Boca Raton, FL, 1990.
[27] R. F. M. deAlmeida, A. Fedorov, M. Prieto, Biophys. J. 2003, 85, 2406.

[28] a) J. Katsaras, V. A. Raghunathan, E. J. Dufourc, J. Dufourcq, Biochemistry 1995, 34, 4684; b) D. Marsh, Chem. Phys. Lipids 2012, 165,59 .

[29] N. Albon, J. Chem. Phys. 1983, 79, 469. 